\title{
Plasma, Cryoprecipitated Reduced
}

National Cancer Institute

\section{Source}

National Cancer Institute. Plasma, Cryoprecipitated Reduced. NCI Thesaurus. Code C133345.

Plasma from which cryoprecipitated antihemophilic factor (AHF) has been removed. 